\title{
The Capacity of APOB-Depleted Plasma in Inducing ATP-Binding Cassette A1/G1-Mediated Macrophage Cholesterol Efflux-But Not Gut Microbial-Derived Metabolites-Is Independently Associated with Mortality in Patients with ST-Segment Elevation Myocardial Infarction
}

\author{
Marina Canyelles 1,2,3,4 (D) Álvaro García-Osuna ${ }^{2}$ (D) Alexandra Junza ${ }^{3,5}$, Oscar Yanes ${ }^{3,5}$ (D) Núria Puig ${ }^{1,4}$, \\ Jordi Ordóñez-Llanos ${ }^{2,6}{ }^{(\mathbb{D}}$, Alessandro Sionis ${ }^{7,8}$, Jordi Sans-Roselló ${ }^{7}$, Aitor Alquézar-Arbé ${ }^{9}$, David Santos ${ }^{1,3}$ \\ Noemi Rotllan ${ }^{1,3} \mathbb{D}^{D}$, Josep Julve ${ }^{1,3}(\mathbb{D}$, Mireia Tondo $2,3, *,+(\mathbb{D}$, Joan Carles Escolà-Gil $1,3, *,+(\mathbb{D})$ \\ and Francisco Blanco-Vaca $2,3,4, *,+(\mathbb{D}$
}

Citation: Canyelles, M.; García-Osuna, Á.; Junza, A.; Yanes, O.; Puig, N.; Ordóñez-Llanos, J.; Sionis, A.; Sans-Roselló, J.; Alquézar-Arbé, A.; Santos, D.; et al. The Capacity of APOB-Depleted Plasma in Inducing ATP-Binding Cassette A1/G1-Mediated

Macrophage Cholesterol Efflux-But

Not Gut Microbial-Derived

Metabolites-Is Independently

Associated with Mortality in Patients with ST-Segment Elevation

Myocardial Infarction. Biomedicines 2021, 9, 1336. https://doi.org/ 10.3390/biomedicines9101336

Academic Editor: Stefano Bellosta

Received: 10 August 2021

Accepted: 22 September 2021

Published: 27 September 2021

Publisher's Note: MDPI stays neutral with regard to jurisdictional claims in published maps and institutional affiliations.

Copyright: (c) 2021 by the authors. Licensee MDPI, Basel, Switzerland. This article is an open access article distributed under the terms and conditions of the Creative Commons Attribution (CC BY) license (https:// creativecommons.org/licenses/by/ $4.0 /)$
1 Institut de Recerca de l'Hospital Santa Creu i Sant Pau, Institut d'Investigacions Biomèdiques, IIB Sant Pau, 08041 Barcelona, Spain; mcanyelles@santpau.cat (M.C.); npuigg@santpau.cat (N.P.);

daymer11@hotmail.com (D.S.); NRotllanV@santpau.cat (N.R.); JJulve@santpau.cat (J.J.)

2 Department of Clinical Biochemistry, Hospital de la Santa Creu i Sant Pau, IIB Sant Pau, 08041 Barcelona, Spain; agarciao@santpau.cat (Á.G.-O.); jordonez1952@gmail.com (J.O.-L.)

3 CIBER de Diabetes y Enfermedades Metabólicas Asociadas (CIBERDEM), 28029 Madrid, Spain; alexandra.junza@urv.cat (A.J.); oscar.yanes@urv.cat (O.Y.)

4 Department de Bioquímica i Biologia Molecular, Universitat Autònoma de Barcelona, 08041 Barcelona, Spain 5 Metabolomics Platform, Department of Electronic Engineering, Universitat Rovira i Virgili, 43204 Reus, Spain 6 Fundació per la Bioquímica i la Patologia Molecular, 08041 Barcelona, Spain

7 Servei de Cardiología, Hospital Santa Creu i Sant Pau, 08041 Barcelona, Spain; asionis@santpau.cat (A.S.); jordisansrosello@hotmail.com (J.S.-R.)

CIBER de Enfermedades Cardiovasculares (CIBERCV), 28029 Madrid, Spain

9 Servei d'Urgències, Hospital Santa Creu i Sant Pau, 08041 Barcelona, Spain; aalquezar@santpau.cat

* Correspondence: mtondo@santpau.cat (M.T.); jescola@santpau.cat (J.C.E.-G.); fblancova@santpau.cat (F.B.-V.)

+ These authors contributed equally to this work.

Abstract: Impaired HDL-mediated macrophage cholesterol efflux and higher circulating concentrations of trimethylamine $\mathrm{N}$-oxide (TMAO) levels are independent risk factors for cardiovascular mortality. The TMAO precursors, $\gamma$-butyrobetaine $(\gamma \mathrm{BB})$ and Trimethyllysine (TML), have also been recently associated with cardiovascular death, but their interactions with HDL-mediated cholesterol efflux remain unclear. We aimed to determine the associations between APOB depleted plasmamediated macrophage cholesterol efflux and plasma TMAO, $\gamma \mathrm{BB}$, and TML concentrations and explore their association with two-year follow-up mortality in patients with acute ST-elevation myocardial infarction (STEMI) and unstable angina (UA). Baseline and ATP-binding cassette transporter ABCA1 and ABCG1 (ABCA1/G1)-mediated macrophage cholesterol efflux to APOB-depleted plasma was decreased in patients with STEMI, and the latter was further impaired in those who died during follow-up. Moreover, the circulating concentrations of TMAO, $\gamma \mathrm{BB}$, and TML were higher in the deceased STEMI patients when compared with the STEMI survivors or UA patients. However, after statistical adjustment, only ABCA1/G1-mediated macrophage cholesterol efflux remained significantly associated with mortality. Furthermore, neither the TMAO, $\gamma \mathrm{BB}$, nor TML levels altered the HDL-mediated macrophage cholesterol efflux in vitro. We conclude that impaired ABCA1/G1-mediated macrophage cholesterol efflux is independently associated with mortality at follow-up in STEMI patients.

Keywords: HDL-mediated efflux; macrophages; trimethylamine N-oxide; trimethyllysine; myocardial infarction 


\section{Introduction}

The ability of high-density lipoprotein (HDL) particles to stimulate cholesterol efflux from macrophage foam cells, the first step of reverse cholesterol transport (RCT), is one major recognized HDL cardioprotective function [1]. Macrophage cholesterol efflux to HDL occurs via different mechanisms, termed simple aqueous diffusion, facilitated by scavenger receptor class $\mathrm{B}$ type I (SR-BI) and active transport induced by the transmembrane protein ATP-binding cassette transporter ABCA1 and ABCG1 (ABCA1/G1)-mediated pathways. The importance of HDL-mediated cholesterol efflux in atheroprotection emerged from a study reporting a strong inverse association between the ex vivo cholesterol efflux capacity of APOB-depleted serum, measured in cultured mouse macrophage foam cells, and the carotid intima-media thickness and likelihood of angiographical-defined coronary artery disease [2]. Despite another report found that higher cholesterol efflux was associated with an increased prospective risk of a composite cardiovascular endpoint of incident myocardial infarction (MI), stroke, or death [3], two subsequent studies confirmed an inverse association of HDL-mediated cholesterol efflux and incident coronary heart disease risk independent of HDL cholesterol (HDL-C) concentrations [4,5]. The HDL-mediated cholesterol efflux ability in mouse macrophage foam cells was further found inversely associated with cardiovascular mortality in patients with chronic coronary artery disease [6-8]. Moreover, a recent report showed that the serum cholesterol efflux capacity measured in human macrophages was also a strong predictor of all-cause mortality after a MI, but the study did not find any association with HDL-mediated efflux or ABCA1-dependent and SR-BI-mediated serum efflux capacities [9].

Trimethylamine N-oxide (TMAO), a gut microbial-derived metabolite of choline and L-carnitine, among other dietary precursors, has been associated with major adverse cardiovascular events [9-11]. Higher plasma TMAO concentrations have also been associated with higher mortality risk in patients with heart failure $[12,13]$ and chronic kidney disease [14]. Two recent reports investigated the potential of TMAO concentrations in risk stratification after an MI. The two studies found that TMAO was a predictive biomarker of all-cause [15] and cardiovascular death [16], but only one of the studies found a role of TMAO as a predictor of reinfarction [15]. Finally, high levels of TMAO in plasma were also independently correlated with plaque rupture in patients with ST-segment elevation MI (STEMI) [17]. Beyond TMAO, $\gamma$-butyrobetaine $(\gamma \mathrm{BB})$, a major gut microbial metabolite produced from dietary L-carnitine, and its precursor Trimethyllysine (TML), have been associated with cardiovascular mortality in patients with carotid atherosclerosis [18]. TML and TMAO were also associated with major adverse cardiovascular events and all-cause mortality amongst patients presenting with acute coronary syndrome [11]. However, only TML (and not TMAO nor $\gamma$-BB) was found to predict acute MI in patients who underwent coronary angiography [19].

A potential link among TMAO, liver, and intestine cholesterol homeostasis and HDL function has been described [20]. TMAO may enhance foam cell formation by upregulating macrophage scavenger receptors, but, in experimental models, it has also been shown to deregulate enterohepatic cholesterol and bile acid metabolism and impairs macrophage RCT [21,22]. However, it has also been described that TMAO enhances macrophage cholesterol efflux, at least in part due to the upregulation of $A b c a 1$ and $A b c g 1$ in peritoneal mouse macrophages [22]. Nonetheless, some studies have also reported TMAO downregulating $A b c a 1$ gene expression in murine macrophages $[23,24]$.

Altogether, although the prognostic value of macrophage cholesterol efflux and TMAO in predicting cardiovascular mortality in patients with MI has been reported, the association of both parameters together with other TMAO precursors has never been explored. Here, we aimed to evaluate the associations among HDL-mediated macrophage cholesterol efflux, TMAO, $\gamma \mathrm{BB}$, and TML with cardiovascular mortality in patients in the acute phase of STEMI and test whether the gut microbial-derived metabolites modulate the associations between HDL-mediated macrophage cholesterol efflux and mortality. 


\section{Experimental Section}

\subsection{Study Population and Data Collection}

Blood samples from patients with STEMI (diagnosed according to the principles of the Universal Definition of Myocardial Infarction) [25,26] were obtained from a retrospective study that included 253 patients consecutively admitted to the Hospital de la Santa Creu i Sant Pau. The patients were followed for two years for cardiovascular adverse events, allcause death, and hospital readmissions via telephone interview and/or electronic medical record review. The primary outcome of this study was cardiovascular death defined as death by cardiogenic shock, fatal MI, or heart failure. We selected a sub-sample of STEMI patients: 35 who died during admission or during follow-up and 36 who survived. In all STEMI patients, the Global Registry of Acute Coronary Events (GRACE 2.0) risk score was calculated [27]. A subgroup of 33 patients with suggested symptoms of MI but highsensitive cardiac troponin T (hs-cTnT) concentrations below the cut-off used to define myocardial damage ( $14 \mathrm{ng} / \mathrm{L}$ ) or not reaching an hourly increase $>3.0 \mathrm{ng} / \mathrm{L}$ during threehour serial sampling was diagnosed with unstable angina (UA) and used as the control group. All the subjects gave informed consent. The study was performed in accordance with the ethical principles set forth in the Declaration of Helsinki. UA and STEMI survivors were matched with the deceased STEMI group for age, sex, body mass index, arterial hypertension, and diabetes mellitus and smoking status.

\subsection{Blood Samples and Biochemical Measurements}

Blood samples were collected in EDTA anticoagulated tubes for the UA patients upon their admission and immediately before the angiographic procedure performed at the time of event in the STEMI patients. Plasma was obtained via centrifugation (10 min, at $10,000 \times g$ ), hs-cTnT was measured in STAT-mode (Cobas e601, Roche Diagnostics, Basel, Switzerland), and aliquots were stored at $-80{ }^{\circ} \mathrm{C}$ until analysis. Triglyceride, low-density lipoprotein cholesterol (LDL-C), and C-reactive protein (CRP) levels were measured enzymatically using commercial kits adapted for a COBAS 6000 autoanalyzer (Roche Diagnostics, Basel, Switzerland). Plasma HDL-C levels were measured after the precipitation of apolipoprotein (APO) B-containing lipoprotein particles with $0.44 \mathrm{mmol} / \mathrm{L}$ phosphotungstic acid (Merck, Darmstadt, Germany) and $20 \mathrm{mmol} / \mathrm{L}$ magnesium chloride (Sigma-Aldrich, Madrid, Spain). Plasma creatinine, to estimate the glomerular filtration rate (eGFR) (using the CKD-EPI formula), was analyzed with an Architect c16000 analyzer (Abbott Diagnostic, Chicago, IL, USA).

\subsection{Ex Vivo and In Vitro Cholesterol Efflux Capacity}

The ex vivo cellular cholesterol efflux was determined using TopFluor-cholesterol, a fluorescent cholesterol probe in which the cholesterol molecule is linked to a boron dipyrromethene difluoride (BODIPY) moiety (Avanti Polar Lipids, Alabaster, AL, USA). J774A.1 cells $\left(7.5 \times 10^{4} /\right.$ well $)$ were seeded in 48 -well plates and allowed to grow for $24 \mathrm{~h}$ in a DMEM-supplemented medium. Next, macrophages were labeled for $1 \mathrm{~h}$ in a high-glucose DMEM medium (Lonza, Waltham, MA, USA) containing $0.125 \mathrm{mmol} / \mathrm{L}$ total cholesterol, where the fluorescent cholesterol accounted for $20 \%$ of the total cholesterol complexed with $10 \mathrm{mmol} / \mathrm{L}$ methyl- $\beta$-cyclodextrin (Sigma-Aldrich, Madrid, Spain), as reported in [28]. The labeled cells were subsequently equilibrated for $18 \mathrm{~h}$ with DMEM containing $0.2 \%$ fatty-acid free BSA (Sigma-Aldrich, Madrid, Spain) and then incubated for $24 \mathrm{~h}$ with $1 \%$ APOB-depleted plasma in a Roswell Park Memorial Institute (RPMI) medium. The acylCoA cholesterol acyltransferase (ACAT) inhibitor, Sandoz 58-035 (5 $\mu \mathrm{mol} / \mathrm{L}$; Sigma Aldrich, Madrid, Spain), was present during the whole experimental procedure. This experiment was performed under baseline conditions and also under experimental settings mainly stimulating the concerted ABCA1/ABCG1-dependent efflux pathways by pre-treating the cells with $2 \mu \mathrm{mol} / \mathrm{L}$ of the liver $\mathrm{X}$ receptor agonist, T0901317 (Cayman Chemicals, Ann Arbor, MI, USA), during the equilibration process or, alternatively, under experimental settings that only stimulated the ABCA1-dependent pathway by pre-treating the cells with 
$0.3 \mathrm{mmol} / \mathrm{L}$ of cyclic adenosine monophosphate (cAMP, Sigma-Aldrich, Madrid, Spain). The fluorescence intensity was then measured in the medium using the microplate reader, Synergy HT (BioTek Instruments, Winooski, VT, USA), at $\lambda E x / E m=485 / 530 \mathrm{~nm}$. The cells were solubilized with $1 \%$ cholic acid and mixed on a plate shaker for $4 \mathrm{~h}$ at room temperature, and the fluorescence intensity was quantified. The cholesterol efflux capacity was calculated according to the formula: [media fluorescence/(media fluorescence + cells fluorescence) $] \times 100$. All conditions were run in duplicate. Values were normalized using an APOB-depleted plasma pool within each efflux assay.

In the in vitro study, macrophages were pre-treated with $2 \mu \mathrm{mol} / \mathrm{L}$ of T0901317 and then incubated with $0,5,20$, and $40 \mu \mathrm{M}$ of TMAO, $0,0.5$, and $2 \mu \mathrm{M}$ of $\gamma \mathrm{BB}$ or $0,0.5,1$, and $2 \mu \mathrm{M}$ TML (Sigma-Aldrich, Madrid, Spain) for $24 \mathrm{~h}$ with $1 \%$ of and APOB-depleted plasma pool. The pool of plasma samples from normolipemic patients was obtained from the Clinical Laboratory of Hospital de la Santa Creu i Sant Pau. The cholesterol efflux capacity was determined as described above and expressed as $\%$ at $24 \mathrm{~h}$.

\subsection{Plasma TMAO, $\gamma B B$ and TML Determinations}

First, $25 \mu \mathrm{L}$ of human plasma was mixed and vigorously vortexed for $20 \mathrm{~s}$ with $300 \mu \mathrm{L}$ of acetonitrile: methanol: water $(5: 4: 1 ; v: v: v)$ containing two internal standards (IS) for quantification. The internal standards used were $d_{3}$-methylcarnitine $\left(\mathrm{d}_{3}\right.$-MeCar) to quantify the $\gamma \mathrm{BB}$ and TMAO concentrations and ${ }^{13} \mathrm{C}_{3}$-TML to quantify the TML-both at $5 \mathrm{ppm}$. After $30 \mathrm{~min}$ of re-equilibration on ice, the samples were centrifuged for $10 \mathrm{~min}$ at $25,100 \times g$ and $4{ }^{\circ} \mathrm{C}$, and the supernatant was transferred into the vial prior to the LC-MS analysis. To quantify the analytes in the plasma, matrix-matched calibration curves were prepared using a human plasma pool spiked with standards. The concentration ranges of the calibration curves were $0-250 \mu \mathrm{M}, 0-25 \mu \mathrm{M}$ and $0-20 \mu \mathrm{M}$ for the TMAO, GBB and TML, respectively.

The extracts were analyzed using an ultra-high performance LC system coupled with a 6490 triple-quadrupole mass spectrometer (QqQ, Agilent Technologies, Santa Clara, CA, USA) with an electrospray ion source (LC-ESI-QqQ) working in positive mode. An ACQUITY UPLC BEH HILIC column $(1.7 \mathrm{~mm}, 2.1 \times 150 \mathrm{~mm}$, Waters $)$ and a gradient mobile phase consisting of water with $50 \mathrm{mM}$ ammonium acetate (phase A) and acetonitrile (phase B) were used for chromatographic separation. The gradient was as follows: isocratic for $30 \mathrm{~s}$ at $75 \% \mathrm{~B}$, from 0.5 to $2 \mathrm{~min}$ decreased to $65 \% \mathrm{~B}$, from 2 to $2.1 \mathrm{~min}$ decreased to $45 \%$ $\mathrm{B}$, from 2.1 to $3.9 \mathrm{~min}$ isocratic at $45 \% \mathrm{~B}$, for $0.1 \mathrm{~min}$ raised to $75 \% \mathrm{~B}$, and, finally, column equilibrated at $75 \%$ B until $5.5 \mathrm{~min}$. The flow of the method was $0.6 \mathrm{~mL} / \mathrm{min}$. Then, $2 \mu \mathrm{L}$ of plasma extract was injected in the LC system. The mass spectrometer parameters were as follows: drying and sheath gas temperatures of $280^{\circ} \mathrm{C}$ and $400{ }^{\circ} \mathrm{C}$, respectively; source and sheath gas flows of 20 and $12 \mathrm{~L} / \mathrm{min}$, respectively; nebulizer flow of 60 psi; capillary voltage of $2500 \mathrm{~V}$; nozzle voltage of $500 \mathrm{~V}$; and iFunnel HRF and LRF of 110 and $80 \mathrm{~V}$, respectively. The QqQ worked in MRM mode using defined transitions. The transitions for TML, ${ }^{13} \mathrm{C}_{3}$-TML (IS), GBB, TMAO, and $\mathrm{d}_{3}-\mathrm{MeCar}(\mathrm{IS})$ and the collision energy $(\mathrm{CE}(\mathrm{V}))$ were: TML $189 \rightarrow 84(17), 189 \rightarrow 130(30) ;{ }^{13} C_{3}$-TML(IS) $192 \rightarrow 84(21), 192 \rightarrow 130(13) ;$ GBB $146 \rightarrow 87(16)$, $146 \rightarrow 60(12) ;$ TMAO $76 \rightarrow 58(16), 76 \rightarrow 59(8) ; d_{3}-M e C a r(I S) ~ 165 \rightarrow 63(16), 165 \rightarrow 103(16)$.

\subsection{Statistical Methods}

Data is presented as the mean \pm standard deviation (SD) for continuous variables and as frequencies and percentages for categorical variables. A chi-square test was used to compare the categorical data between groups. The normality of the data was analyzed using the Kolmogorov-Smirnov and D'Agostino and Pearson omnibus tests. A one-way analysis of variance (ANOVA) test was used to compare the continuous variables, and Tukey's post-test was used for comparing differences among the groups. A Kruskal-Wallis test was used to compare continuous variables not following a Gaussian distribution, and Dunn's post-test was used for comparing differences among the groups. Correlations between variables were analyzed using Pearson's correlation analysis. Multivariate analysis of 
covariance (ANCOVA) was used to explore the associations with mortality, adjusting for potential confounders. The statistical software, IBM SPSS Statistics v23, and GraphPad Prism 6.0 software (GraphPad, San Diego, CA, USA) were used to perform all statistical analyses. A $p$-value $<0.05$ was considered to be statistically significant.

\section{Results}

\subsection{Study Subjects}

The baseline clinical and plasma biochemical characteristics of patients admitted with UA and those with STEMI who survived or died in a two-year follow-up period are shown in Table 1. The triglyceride concentrations did not differ among the three patient groups. HDL-C concentrations were significantly lower in patients with STEMI compared to those with UA but not different between the survivors and deceased STEMI patients. The deceased STEMI patients showed significantly lower LDL-C when compared with the survivors, and the latter presented higher LDL-C concentrations than the UA patients. Logarithmically transformed plasma CRP values were higher in patients with STEMI compared with those in the UA group, and this value was higher in the deceased STEMI patients when compared with the survivors (Table 1). The eGFR was also significantly lower in the deceased STEMI patients when compared with the STEMI survivors and those admitted with UA (Table 1). Also, the GRACE risk score and hs-cTnT were higher in the STEMI deceased patients when compared with the STEMI survivors (Table 1). The percentage of STEMI patients treated with statins was 38\% for survivors and $44 \%$ for deceased $(p=0.811)$.

Table 1. Clinical and biochemical parameters of the studied patients at admission.

\begin{tabular}{|c|c|c|c|c|}
\hline & $\begin{array}{c}\mathrm{UA} \\
n=33\end{array}$ & $\begin{array}{l}\text { STEMI Survivors } \\
\qquad n=36\end{array}$ & $\begin{array}{l}\text { STEMI Deceased } \\
\qquad n=35\end{array}$ & $p$-Value \\
\hline Age (years) & $71.97 \pm 3.45$ & $72.06 \pm 7.3$ & $73.41 \pm 9.86$ & 0.6656 \\
\hline $\operatorname{Sex}(\mathrm{M} / \mathrm{F})$ & $17 / 16$ & $20 / 16$ & $19 / 15$ & 0.923 \\
\hline Body mass index $\left(\mathrm{Kg} / \mathrm{m}^{2}\right)$ & $26.38 \pm 2.86$ & $26.66 \pm 2.06$ & $27.07 \pm 4.07$ & 0.6774 \\
\hline Hypertension $(\%)$ & 56 & 72 & 71 & 0.3525 \\
\hline Diabetes mellitus (\%) & 21 & 25 & 41 & 0.1903 \\
\hline Smoking $(\%)$ & 17 & 25 & 35 & 0.0859 \\
\hline Triglycerides (mmol/L) & $1.07 \pm 0.47$ & $0.97 \pm 0.4$ & $1.12 \pm 0.73$ & 0.5012 \\
\hline HDL-C $(\mathrm{mmol} / \mathrm{L})$ & $1.63 \pm 0.44$ & $1.33 \pm 0.34^{* *}$ & $1.19 \pm 0.31^{* * * *}$ & $<0.0001$ \\
\hline LDL-C $(\mathrm{mmol} / \mathrm{L})$ & $2.21 \pm 0.64$ & $2.81 \pm 1.01^{* *}$ & $2.12 \pm 0.89+\dagger$ & 0.0022 \\
\hline $\log _{10}$ C-reactive protein $(\mathrm{mg} / \mathrm{L})$ & $3.30 \pm 0.63$ & $3.78 \pm 0.71 *$ & $4.42 \pm 0.87^{* * * *}+\dagger$ & $<0.0001$ \\
\hline eGFR $\left(\mathrm{mL} / \mathrm{min} / 1.73 \mathrm{~m}^{2}\right)$ & $77.74 \pm 13.94$ & $69.66 \pm 18.21$ & $51.11 \pm 20.50^{* * * *}$ +†+† & $<0.0001$ \\
\hline GRACE 2.0 risk score & ND & $188.5 \pm 44.23$ & $251.6 \pm 44.35$ t+†† & $<0.0001$ \\
\hline $\log _{10}$ hs-cTnT (ng/L) & $3.90 \pm 0.17$ & $5.36 \pm 0.79^{* * * *}$ & $6.06 \pm 0.72$ †ナ†ナ**** & $<0.0001$ \\
\hline
\end{tabular}

UA: unstable angina; STEMI: ST-segment elevation myocardial infarction; HDL-C and LDL-C: high- and low-density lipoprotein cholesterol; eGFR: estimated glomerular filtration rate by CKD-EPI equation; GRACE 2.0: Global Registry of Acute Coronary Events. ND, nondetermined. Results are presented as mean \pm standard deviation (SD). ${ }^{*} p<0.05^{* *} p<0.01^{* * * *} p<0.0001$ vs. UAt+ $p<0.01++++p<0.0001$ vs. STEMI survivors.

\subsection{ABCA1 and ABCG1-Mediated Macrophage Cholesterol Efflux to APOB-Fepleted Serum Ex Vivo Is Down Regulated in Deceased STEMI Patients}

The ex vivo ability of APOB-depleted plasmas in inducing macrophage cholesterol efflux was evaluated in all the groups under baseline conditions and experimental settings stimulating only the ABCA1 or both the ABCA1/G1 pathways. The baseline macrophage cholesterol efflux was significantly lower in the deceased STEMI patients when compared with the STEMI survivors and those with UA (Figure 1A). The ABCA1-mediated macrophage cholesterol efflux was also decreased in the patients who died when compared with the UA group (Supplementary Materials, Figure S1). This change was also observed after stimulating both the ABCA1/G1-dependent efflux pathways, but, in this case, the macrophage cholesterol efflux was also more decreased in the STEMI survivors when compared with the UA patients (Figure 1B). 
A

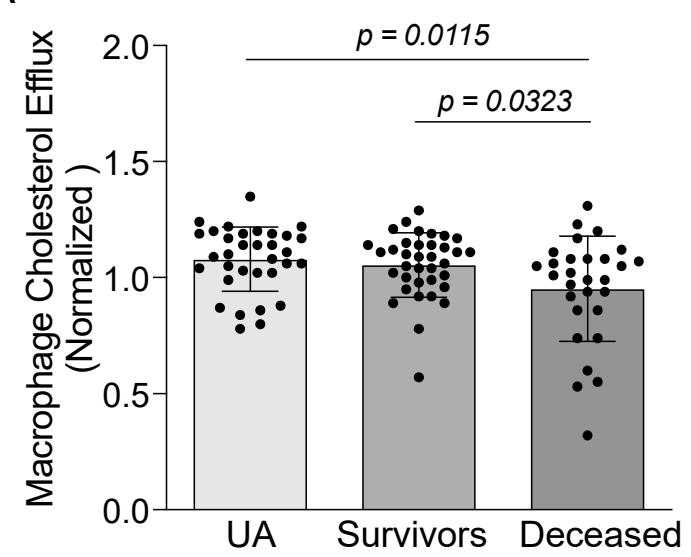

B

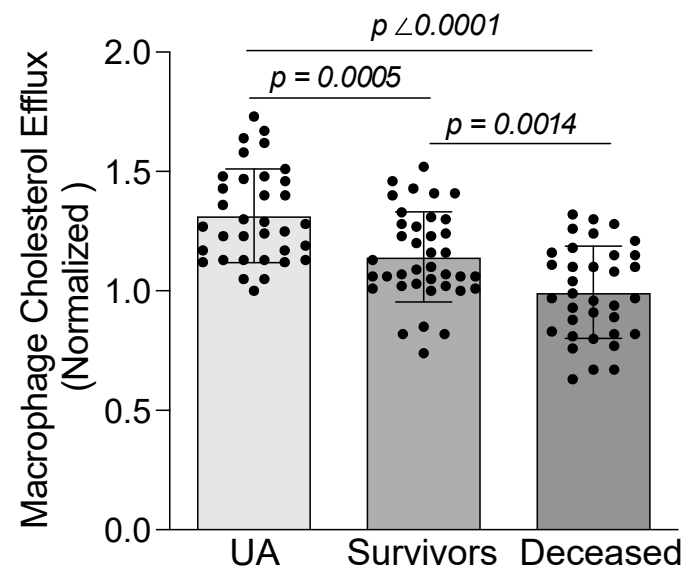

Figure 1. Macrophage cholesterol efflux is impaired in STEMI patients who died within a two-year follow-up period under baseline conditions (ANOVA $p$ value $=0.0096)(A)$ and after activation of macrophage ABCA1/G1-dependent pathways by the LXR agonist, T090137 (ANOVA $p$ value < 0.0001) (B). Values are represented as mean \pm SD for UA $(n=33)$, STEMI survivors $(n=36)$ and deceased STEMI $(n=35)$.

\subsection{Circulating Levels of TMAO, $\gamma B B$, and TML Are Increased in STEMI Patients Who Died during Follow-Up}

Plasma concentrations of TMAO, $\gamma \mathrm{BB}$ and TML were assessed in the same samples that APOB-depleted plasma-mediated cholesterol efflux was determined. The levels of these metabolites were significantly higher in the deceased STEMI patients compared to the STEMI survivors and UA patients (Figure 2).

A

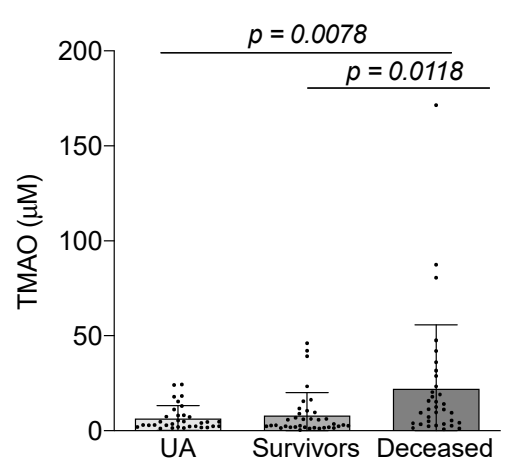

B

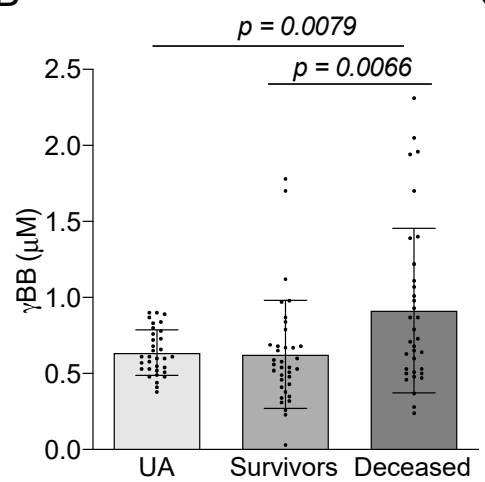

C

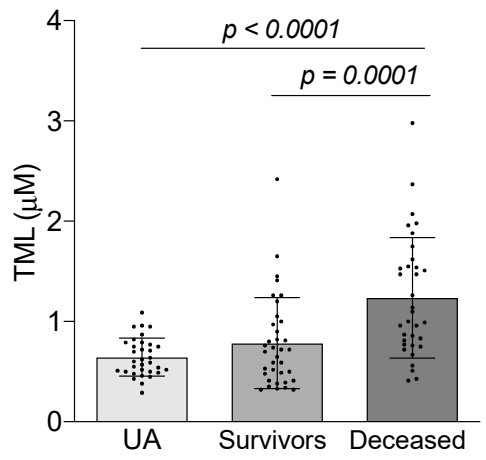

Figure 2. Plasma TMAO (ANOVA $p$ value $=0.0040)(\mathbf{A}) ; \gamma \mathrm{BB}($ ANOVA $p$ value $=0.0029)(\mathbf{B})$ and TML (ANOVA $p$ value $<0.0001)(C)$ levels were higher in deceased STEMI patients than in the other two groups. Values are represented as mean \pm SD in UA patients $(n=33)$, STEMI survivors $(n=36)$, and deceased STEMI patients $(n=35)$.

\subsection{ABCA1/G1-Mediated Macrophage Cholesterol Efflux Is Independently Associated with Mortality}

We further evaluated the association between APOB-depleted plasma-mediated macrophage cholesterol efflux and mortality in these patients after adjusting for the main covariates that were significantly correlated with macrophage cholesterol efflux, including HDL-C, TMAO, and eGFR (all with $p<0.05$ ). The association between the ABCA1/G1mediated macrophage cholesterol efflux and death remained significant after adjusting for these parameters in (a) of (Table 2). However, both the baseline and ABCA1-mediated macrophage cholesterol efflux were not significantly associated with death following adjustment for these factors (Supplementary Materials Table S1). Furthermore, the ABCA1/G1mediated macrophage cholesterol efflux association with death also remained significant 
when TML (adjusted R-squared $=0.469 ; p=0.009$ ) or $\gamma \mathrm{BB}$ was included in the analyses (adjusted R-squared $=0.483 ; p=0.009$ ). Next, we further adjusted for other covariates that correlated with the ABCA1/G1-mediated macrophage cholesterol efflux $(p<0.05)$ which strongly predict STEMI mortality, such as hs-cTnT, GRACE score, HDL-C and CRP. The association of the ABCA1/G1-mediated macrophage cholesterol efflux with death still remained significant after adjustment in (b) of (Table 2).

Table 2. Analysis of covariance (ANCOVA) of ABCA1/G1-mediated macrophage cholesterol efflux at $24 \mathrm{~h}$ adjusted for (a) HDL-C, eGFR and TMAO that significantly correlated with the dependent variable or (b) hs-cTnT, GRACE score, HDL-C and CRP, factors strongly associated with mortality.

\begin{tabular}{|c|c|c|c|c|c|}
\hline (a) & & & & & \\
\hline Source & SS & Df & $\begin{array}{l}\text { Mean } \\
\text { Square }\end{array}$ & $\mathbf{F}$ & $p$ Value \\
\hline Corrected model & $2.610 *$ & 5 & 0.522 & 18.403 & 0.000 \\
\hline Intercept & 2.287 & 1 & 2.287 & 80.636 & 0.000 \\
\hline HDL-C & 0.776 & 1 & 0.776 & 27.371 & 0.000 \\
\hline eGFR & 0.043 & 1 & 0.043 & 1.509 & 0.222 \\
\hline TMAO & 0.000 & 1 & 0.000 & 0.011 & 0.917 \\
\hline Death & 0.331 & 2 & 0.166 & 5.838 & 0.004 \\
\hline Error & 2.666 & 94 & 0.028 & & \\
\hline Total & 139.441 & 100 & & & \\
\hline Corrected total & 5.275 & 99 & & & \\
\hline \multicolumn{6}{|l|}{$\begin{array}{c}* \text { R-squared }=0.495 \\
\text { (adjusted R-squared }=0.468 \text { ) }\end{array}$} \\
\hline \multicolumn{6}{|l|}{ (b) } \\
\hline Source & SS & Df & $\begin{array}{l}\text { Mean } \\
\text { Square }\end{array}$ & $\mathbf{F}$ & $p$ Value \\
\hline Corrected model & $0.755 *$ & 5 & 0.151 & 4.652 & 0.001 \\
\hline Intercept & 0.119 & 1 & 0.119 & 3.674 & 0.060 \\
\hline hs-cTnT & 0.034 & 1 & 0.034 & 1.054 & 0.309 \\
\hline GRACE score & 0.007 & 1 & 0.007 & 0.213 & 0.646 \\
\hline HDL-C & 0.440 & 1 & 0.440 & 13.553 & 0.000 \\
\hline CRP & 0.000 & 1 & 0.000 & 0.008 & 0.931 \\
\hline Death & 0.188 & 1 & 0.188 & 5.804 & 0.019 \\
\hline Error & 2.012 & 62 & 0.032 & & \\
\hline Total & 81.888 & 68 & & & \\
\hline Corrected total & 2.767 & 67 & & & \\
\hline $\begin{array}{l}\quad * \text { R-squared }=0.114 \\
\text { (adjusted R-squared }=0.058 \text { ) }\end{array}$ & & & & & \\
\hline
\end{tabular}

Also, we evaluated the association between gut microbial-derived metabolites and mortality in these patients after adjusting for the main determinant of these metabolites, eGFR, and also for the main parameter of interest, the ABCA1/G1-mediated macrophage cholesterol efflux. The association between TMAO (adjusted R-squared $=0.099 ; p=0.088$ ) or $\gamma \mathrm{BB}$ (adjusted R-squared $=0.104 ; p=0.123$ ) with death did not remain significant after adjusting for these parameters. In contrast, TML retained its independent association with death after adjustment for these parameters in (a) of (Table 3). However, the independent association between TML and mortality did not persist after adjustment for hs-cTnT, GRACE score and CRP in (b) of (Table 3).

\subsection{TMAO, $\gamma B B$, and TML Did Not Affect APOB-Depleted Plasma-Mediated Macrophage Cholesterol Efflux In Vitro}

Finally, we determined the effects of TMAO, $\gamma \mathrm{BB}$, and TML on the capacity of an APOB-depleted plasma pool to induce macrophage cholesterol efflux in vitro. For this purpose, the macrophages were incubated with increasing concentrations of all metabolites 
during the efflux period of $24 \mathrm{~h}$. Importantly, TMAO, $\gamma \mathrm{BB}$ and TML did not modify the ABCA1/G1-mediated macrophage cholesterol efflux to an APOB-depleted plasma pool (Figure $3 \mathrm{~A}-\mathrm{C}$ ).

Table 3. Analysis of ANCOVA of circulating TML levels adjusted for (a) eGFR and normalized ABCA1/G1-mediated efflux or (b) hs-cTnT, GRACE score and CRP.

\begin{tabular}{|c|c|c|c|c|c|}
\hline \multicolumn{6}{|l|}{ (a) } \\
\hline Source & SS & $\mathrm{df}$ & $\begin{array}{l}\text { Mean } \\
\text { Square }\end{array}$ & F & $p$ Value \\
\hline Corrected model & $7.423 *$ & 4 & 1.856 & 10.054 & 0.000 \\
\hline Intercept & 4.155 & 1 & 4.155 & 22.512 & 0.000 \\
\hline eGFR & 1.415 & 1 & 1.415 & 7.665 & 0.007 \\
\hline $\begin{array}{c}\text { Normalized } \\
\text { ABCA1/G1-mediated efflux }\end{array}$ & 0.002 & 1 & 0.002 & 0.011 & 0.917 \\
\hline Death & 1.934 & 2 & 0.967 & 5.239 & 0.007 \\
\hline Error & 17.535 & 95 & 0.185 & & \\
\hline Total & 103.990 & 100 & & & \\
\hline $\begin{array}{c}\text { Corrected total } \\
* \text { R Squared }=0.297 \\
\text { (Adjusted R Squared }=0.268 \text { ) }\end{array}$ & 24.958 & 99 & & & \\
\hline \multicolumn{6}{|l|}{ (b) } \\
\hline Source & SS & $\mathrm{df}$ & $\begin{array}{l}\text { Mean } \\
\text { Square }\end{array}$ & $\mathbf{F}$ & $p$ Value \\
\hline Corrected model & $5.948 *$ & 4 & 1.487 & 5.554 & 0.001 \\
\hline Intercept & 0.051 & 1 & 0.051 & 0.191 & 0.663 \\
\hline hs-cTnT & 0.005 & 1 & 0.005 & 0.018 & 0.894 \\
\hline GRACE score & 1.733 & 1 & 1.733 & 6.471 & 0.013 \\
\hline CRP & $1.455 \times 10^{-5}$ & 1 & $1.455 \times 10^{-5}$ & 0.000 & 0.994 \\
\hline Death & 0.596 & 1 & 0.596 & 2.224 & 0.141 \\
\hline Error & 17.137 & 64 & 0.268 & & \\
\hline Total & 94.710 & 69 & & & \\
\hline $\begin{array}{c}\text { Corrected total } \\
* \text { R-squared }=0.258 \\
\text { (adjusted R-squared }=0.211 \text { ) }\end{array}$ & 23.086 & 68 & & & \\
\hline
\end{tabular}

A

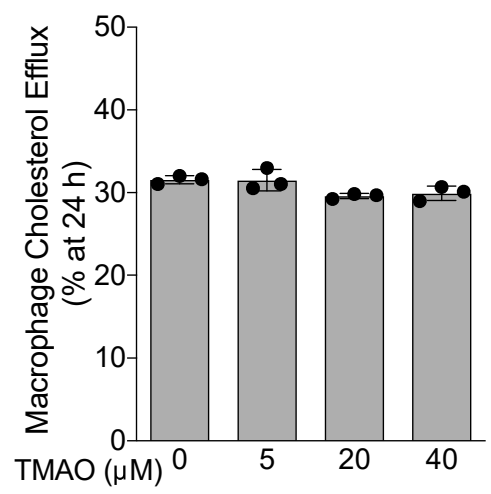

B

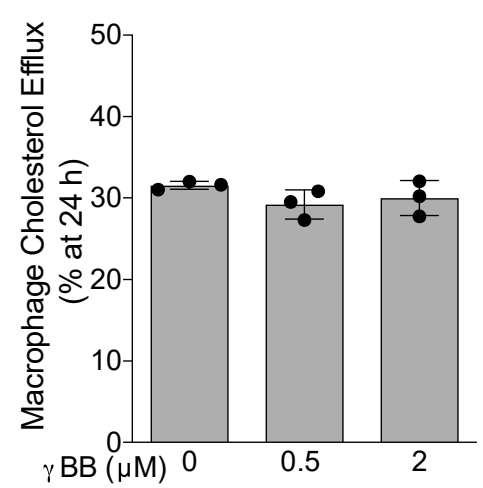

C

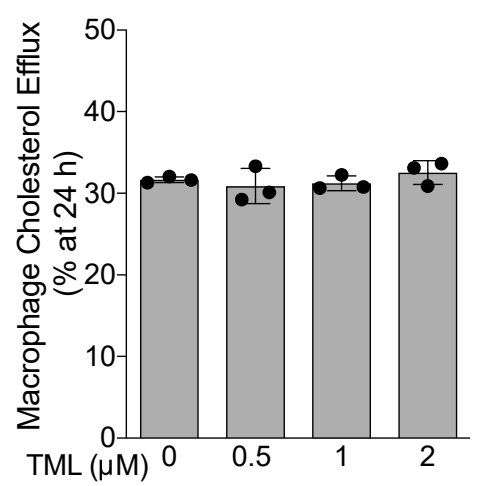

Figure 3. Effects of TMAO, $\gamma \mathrm{BB}$, and TML on ABCA1/G1-mediated macrophage cholesterol efflux to APOB-depleted plasma. The macrophage cholesterol efflux capacity was expressed as $\%$ of fluorescence released at $24 \mathrm{~h}$. TMAO (A), $\gamma \mathrm{BB}$ (B), and TML (C) levels did not affect the macrophage cholesterol efflux to the APOB-depleted plasma under the conditions stimulating the ABCA1/G1-dependent pathways. Kruskal-Wallis and Dunn's post-test did not reveal significant differences among the groups incubated with the gut microbial-derived metabolites versus the control group without TMAO, $\gamma \mathrm{BB}$, and TML. Three independent experiments were performed for each condition. 


\section{Discussion}

Macrophage cholesterol efflux is largely induced by HDL particles. A recent metaanalysis showed that the macrophage cholesterol efflux capacity was inversely associated with the risk of cardiovascular disease and mortality, although the authors also admitted the heterogeneity among the included studies and evidence of publication bias [29]. Part of this heterogeneity could be explained by the methodology used to isolate the HDL particles; many studies have analyzed cholesterol efflux after the chemical precipitation of APOB-containing lipoproteins to isolate HDL particles from serum or plasma, while others used different approaches. Three independent studies showed that the impaired cholesterol efflux capacity of APOB-depleted serum or plasma from mouse macrophages was associated with higher cardiovascular mortality in patients with chronic coronary artery disease [6-8]. In these studies, mouse J774.A1 macrophages were incubated with cAMP to upregulate $A B C A 1$, thereby suggesting the major contribution of a dysfunctional ABCA1-mediated pathway in this cellular model. However, a recent report failed to find any significant association between ABCA1 or SR-BI-mediated cholesterol efflux and the cardiovascular mortality of MI patients, even though it did show that their serum cholesterol efflux capacity was a strong predictor of mortality after a MI [9]. It is noteworthy that this report used human THP-1 macrophages pretreated with acetyl-LDL, which stimulates both ABCA1/G1-mediated efflux [9]. Our work adds to current knowledge that the impaired ABCA1/G1-mediated cholesterol efflux capacity from mouse J774.A1 macrophages to HDL is independently associated with mortality in STEMI patients. Our results reinforce the importance of measuring different components of macrophage cholesterol efflux capacity, integrating both the ABCA1-dependent pathway, which facilitates cholesterol release to lipid-free apoA-I/pre $\beta-H D L$, and the ABCG1-dependent efflux to mature $\alpha$ migrating HDL particles. These assays better mimic the two predominant pathways of cholesterol-loaded macrophages converted into foam cells in atherosclerotic lesions [30].

Our analyses revealed that patients with STEMI who had higher plasma TMAO, $\gamma \mathrm{BB}$, and TML were more likely to experience subsequent death. However, the associations between TMAO or $\gamma \mathrm{BB}$ and death were attenuated when further adjusted for other factors. Despite several reports showing that TMAO independently predicted future cardiovascular adverse events [11,31], not all reports have found independent associations between TMAO levels and cardiovascular outcomes [32-34]. The strong influence of renal function on TMAO levels could explain, at least in part, the inconsistency of TMAO in predicting adverse cardiovascular outcomes [32,33].

TML can be obtained from diet but also from endogenous production via the methylation of lysine residues in histones and, most likely, under the proteolytic degradation of other proteins; furthermore, TML is a poor precursor of TMAO [31]. Of note, the association of TML and death remained significant after adjusting for the main gut microbial-derived metabolite determinants in our study, but it was lost after adjusting for major covariates predicting STEMI mortality [35]. However, other studies have shown TML as an independent prognostic marker of major adverse cardiovascular events and mortality risk $[11,19,31]$. Our results do not necessarily contradict those obtained in patients with suspected stable angina pectoris [19], in subjects without evidence of acute coronary syndrome who underwent elective diagnostic coronary angiography [31], or those who had UA or adjudicated acute coronary syndromes [11]. Therefore, TML levels could provide insight regarding cardiovascular risk assessment but, at least among patients with prior STEMI, were found to not offer additional information for predicting mortality. Overall, our findings do not support the role of TML as a pure specific marker for STEMI mortality.

Previous reports have shown divergent results regarding TMAO-mediated effects on macrophage transporters involved in cholesterol efflux, specifically indicating enhancing [22], neutral [24], or downregulating effects [23]. The present study goes further and demonstrates that TMAO, $\gamma \mathrm{BB}$, and TML, at physiological levels, did not produce significant effects on macrophage cholesterol efflux, indicating that the first step in the RCT process was not affected by these three compounds. Taken together, our data does not 
support any implication of TMAO, $\gamma \mathrm{BB}$, and TML in modulating the association between ABCA1/G1-mediated macrophage cholesterol efflux and STEMI mortality.

The present study has some limitations. The number of patients in our cohort was relatively modest, but it was compensated for the fact that we selected homogeneous and well-characterized patient groups, thus providing enough power to conduct our analyses.

\section{Conclusions}

We demonstrate that reduced ABCA1/G1-mediated macrophage cholesterol efflux is independently associated with mortality in STEMI patients. Consistent with the gradual severity of acute coronary syndrome, STEMI patients who survived presented higher ABCA1/G1-mediated macrophage cholesterol efflux than those who died. TMAO, $\gamma B B$, and TML did not affect either macrophage cholesterol efflux to APOB-depleted plasma or the association between ABCA1/G1-mediated macrophage cholesterol efflux and mortality. These results motivate further research on therapeutic strategies aiming to improve the ABCA1/G1-mediated macrophage cholesterol efflux capacity.

Supplementary Materials: The Supplementary Materials are available online at https://www. mdpi.com/article/10.3390/biomedicines9101336/s1, Figure S1: Macrophage cholesterol efflux after activation of macrophage ABCA1 by cAMP, Table S1: Analysis of ANCOVA of Baseline (a) and ABCA1-mediated (b) macrophage cholesterol efflux at $24 \mathrm{~h}$.

Author Contributions: Conceptualization: M.C., M.T., F.B.-V., J.O.-L., A.S. and J.C.E.-G.; methodology: M.C., N.P., Á.G.-O., A.J., O.Y. and D.S.; formal analysis: M.C. and Á.G.-O.; investigation: M.C., Á.G.-O., A.J., O.Y., J.O.-L., J.S.-R., A.S., A.A.-A., N.R., J.J., M.T., F.B.-V. and J.C.E.-G.; writing-original draft preparation: M.C., M.T., J.C.E.-G. and F.B.-V.; writing-review and editing: Á.G.-O., J.O.-L., N.R., A.S. and J.J; supervision: F.B.-V. and J.C.E.-G.; funding acquisition: M.C., N.R., M.T., F.B.-V. and J.C.E.-G. All authors have read and agreed on the published version of the manuscript.

Funding: This work was partly funded by the Instituto de Salud Carlos III and FEDER "Una manera de hacer Europa" grants PI18-00164 (to F.B.-V. and M.T.), PI19-00136 (to J.C.E-G), Rio Hortega contract CM20/00033 (to M.C), and Fundació per a la Bioquímica Clínica i Patologia Molecular (to M.C.). This work was funded by Ministerio de Ciencia, Innovación y Universidades (PID2019-104367RB100), and the Subprograma Ramón y Cajal (RYC-201722879) to N.R. CIBERDEM and CIBERCV are initiatives of the Instituto de Salud Carlos III.

Institutional Review Board Statement: The study was conducted according yo the guidelines of the Declaration of Helsinki, and approved by the Ethical Comitee of Hospital de la Santa Creu I Sant Pau (protocol code IIBS-TRO-2015-61, 4 December 2015).

Informed Consent Statement: Informed consent was obtained from all subjects involved in the study.

Data Availability Statement: The data that support the findings of this study will be available to other researchers upon reasonable request.

Conflicts of Interest: The authors declare no conflict of interest.

\section{References}

1. Rohatgi, A.; Westerterp, M.; von Eckardstein, A.; Remaley, A.; Rye, K.-A. HDL in the 21st Century: A Multifunctional Roadmap for Future HDL Research. Circulation 2021, 143, 2293-2309. [CrossRef]

2. Khera, A.V.; Cuchel, M.; De La Llera-Moya, M.; Rodrigues, A.; Burke, M.F.; Jafri, K.; French, B.C.; Phillips, J.A.; Mucksavage, M.L.; Wilensky, R.L.; et al. Cholesterol Efflux Capacity, High-Density Lipoprotein Function, and Atherosclerosis. N. Engl. J. Med. 2011, 364, 127-135. [CrossRef] [PubMed]

3. Li, X.-M.; Tang, W.H.W.; Mosior, M.K.; Huang, Y.; Wu, Y.; Matter, W.; Gao, V.; Schmitt, D.; DiDonato, J.A.; Fisher, E.; et al. Paradoxical Association of Enhanced Cholesterol Efflux with Increased Incident Cardiovascular Risks. Arterioscler. Thromb. Vasc. Biol. 2013, 33, 1696-1705. [CrossRef] [PubMed]

4. Rohatgi, A.; Khera, A.; Berry, J.D.; Givens, E.G.; Ayers, C.R.; Wedin, K.E.; Neeland, I.J.; Yuhanna, I.S.; Rader, D.R.; De Lemos, J.A.; et al. HDL Cholesterol Efflux Capacity and Incident Cardiovascular Events. N. Engl. J. Med. 2014, 371, 2383-2393. [CrossRef] [PubMed] 
5. Saleheen, D.; Scott, R.; Javad, S.; Zhao, W.; Rodrigues, A.; Picataggi, A.; Lukmanova, D.; Mucksavage, M.L.; Luben, R.; Billheimer, J.; et al. Association of HDL cholesterol efflux capacity with incident coronary heart disease events: A prospective case-control study. Lancet Diabetes Endocrinol. 2015, 3, 507-513. [CrossRef]

6. $\quad$ Ritsch, A.; Scharnagl, H.; Marz, W. HDL cholesterol efflux capacity and cardiovascular events. N. Engl. J. Med. 2015, 372, 1870-1871.

7. Liu, C.; Zhang, Y.; Ding, D.; Li, X.; Yang, Y.; Li, Q.; Zheng, Y.; Wang, D.; Ling, W. Cholesterol efflux capacity is an independent predictor of all-cause and cardiovascular mortality in patients with coronary artery disease: A prospective cohort study. Atherosclerosis 2016, 249, 116-124. [CrossRef]

8. Ritsch, A.; Duerr, A.; Kahler, P.; Hunjadi, M.; Stojakovic, T.; Silbernagel, G.; Scharnagl, H.; Kleber, M.E.; März, W. Cholesterol Efflux Capacity and Cardiovascular Disease: The Ludwigshafen Risk and Cardiovascular Health (LURIC) Study. Biomedicines 2020, 8, 524. [CrossRef]

9. Guerin, M.; Silvain, J.; Gall, J.; Darabi, M.; Berthet, M.; Frisdal, E.; Hauguel-Moreau, M.; Zeitouni, M.; Kerneis, M.; Lattuca, B.; et al. Association of Serum Cholesterol Efflux Capacity with Mortality in Patients with ST-Segment Elevation Myocardial Infarction. J. Am. Coll. Cardiol. 2018, 72, 3259-3269. [CrossRef] [PubMed]

10. Li, X.S.; Obeid, S.; Klingenberg, R.; Gencer, B.; Mach, F.; Räber, L.; Windecker, S.; Rodondi, N.; Nanchen, D.; Muller, O.; et al. Gut microbiota-dependent trimethylamine $\mathrm{N}$-oxide in acute coronary syndromes: A prognostic marker for incident cardiovascular events beyond traditional risk factors. Eur. Heart J. 2017, 38, 814-824. [CrossRef] [PubMed]

11. Li, X.S.; Obeid, S.; Wang, Z.; Hazen, B.J.; Li, L.; Wu, Y.; Hurd, A.G.; Gu, X.; Pratt, A.; Levison, B.S.; et al. Trimethyllysine, a trimethylamine $\mathrm{N}$-oxide precursor, provides near- and long-term prognostic value in patients presenting with acute coronary syndromes. Eur. Heart J. 2019, 40, 2700-2709. [CrossRef] [PubMed]

12. Tang, W.H.; Wang, Z.; Fan, Y.; Levison, B.; Hazen, J.E.; Donahue, L.M.; Wu, Y.; Hazen, S.L. Prognostic value of elevated levels of intestinal microbe-generated metabolite trimethylamine-N-oxide in patients with heart failure: Refining the gut hypothesis. $J$. Am. Coll. Cardiol. 2014, 64, 1908-1914. [CrossRef] [PubMed]

13. Suzuki, T.; Heaney, L.M.; Bhandari, S.S.; Jones, D.; Ng, L. TrimethylamineN-oxide and prognosis in acute heart failure. Heart 2016, 102, 841-848. [CrossRef] [PubMed]

14. Tang, W.W.; Wang, Z.; Kennedy, D.J.; Wu, Y.; Buffa, J.A.; Agatisa-Boyle, B.; Li, X.S.; Levison, B.S.; Hazen, S.L. Gut MicrobiotaDependent Trimethylamine N -Oxide (TMAO) Pathway Contributes to Both Development of Renal Insufficiency and Mortality Risk in Chronic Kidney Disease. Circ. Res. 2015, 116, 448-455. [CrossRef] [PubMed]

15. Suzuki, T.; Heaney, L.; Jones, D.; Ng, L. Trimethylamine N-oxide and Risk Stratification after Acute Myocardial Infarction. Clin. Chem. 2017, 63, 420-428. [CrossRef]

16. Gencer, B.; Li, X.S.; Gurmu, Y.; Bonaca, M.P.; Morrow, D.A.; Cohen, M.; Bhatt, D.L.; Steg, P.G.; Storey, R.F.; Johanson, P.; et al. Gut Microbiota-Dependent Trimethylamine N-oxide and Cardiovascular Outcomes in Patients with Prior Myocardial Infarction: A Nested Case Control Study from the PEGASUS-TIMI 54 Trial. J. Am. Heart Assoc. 2020, 9, e015331. [CrossRef]

17. Tan, Y.; Sheng, Z.; Zhou, P.; Liu, C.; Zhao, H.; Song, L.; Li, J.; Zhou, J.; Chen, Y.; Wang, L.; et al. Plasma Trimethylamine N-Oxide as a Novel Biomarker for Plaque Rupture in Patients With ST-Segment-Elevation Myocardial Infarction. Circ. Cardiovasc. Interv. 2019, 12, e007281. [CrossRef]

18. Skagen, K.; Trøseid, M.; Ueland, T.; Holm, S.; Abbas, A.; Gregersen, I.; Kummen, M.; Bjerkeli, V.; Reier-Nilsen, F.; Russell, D.; et al. The Carnitine-butyrobetaine-trimethylamine-N-oxide pathway and its association with cardiovascular mortality in patients with carotid atherosclerosis. Atherosclerosis 2016, 247, 64-69. [CrossRef]

19. Bjørnestad, E.Ø.; Olset, H.; Dhar, I.; Løland, K.; Pedersen, E.K.R.; Svingen, G.F.; Svardal, A.; Berge, R.K.; Ueland, P.M.; Tell, G.S.; et al. Circulating trimethyllysine and risk of acute myocardial infarction in patients with suspected stable coronary heart disease. J. Intern. Med. 2020, 288, 446-456. [CrossRef]

20. Canyelles, M.; Tondo, M.; Cedó, L.; Farràs, M.; Escolà-Gil, J.C.; Blanco-Vaca, F. Trimethylamine N-Oxide: A Link among Diet, Gut Microbiota, Gene Regulation of Liver and Intestine Cholesterol Homeostasis and HDL Function. Int. J. Mol. Sci. 2018, 19, 3228. [CrossRef] [PubMed]

21. Wang, Z.; Klipfell, E.; Bennett, B.J.; Koeth, R.; Levison, B.S.; DuGar, B.; Feldstein, A.E.; Britt, E.B.; Fu, X.; Chung, Y.-M.; et al. Gut Flora Metabolism of Phosphatidylcholine Promotes Cardiovascular Disease. Nature 2011, 472, 57-63. [CrossRef]

22. Koeth, R.A.; Wang, Z.; Levison, B.S.; Buffa, J.A.; Org, E.; Sheehy, B.T.; Britt, E.B.; Fu, X.; Wu, Y.; Li, L.; et al. Intestinal microbiota metabolism of l-carnitine, a nutrient in red meat, promotes atherosclerosis. Nat. Med. 2013, 19, 576-585. [CrossRef]

23. Mohammadi, A.; Gholamhoseyniannajar, A.; Yaghoobi, M.M.; Jahani, Y.; Vahabzadeh, Z. Expression levels of heat shock protein 60 and glucose-regulated protein 78 in response to trimethylamine-N-oxide treatment in murine macrophage J774A.1 cell line. Cell. Mol. Boil. 2015, 61, 94-100.

24. Collins, H.L.; Drazul-Schrader, D.; Sulpizio, A.C.; Koster, P.D.; Williamson, Y.; Adelman, S.J.; Owen, K.; Sanli, T.; Bellamine, A. L-carnitine intake and high trimethylamine N-oxide plasma levels correlate with low aortic lesions in apoE(-/-) transgenic mice expressing CETP. Atherosclerosis 2016, 244, 29-37. [CrossRef]

25. Thygesen, K.; Alpert, J.S.; Jaffe, A.S.; Simoons, M.L.; Chaitman, B.R.; White, H.D. Third Universal Definition of Myocardial Infarction. Circulation 2012, 126, 2020-2035. [CrossRef]

26. Steg, P.G.; James, S.K.; Atar, D.; Badano, L.P.; Lundqvist, C.B.; Borger, M.A.; Di Mario, C.; Dickstein, K.; Ducrocq, G.; FernandezAviles, F.; et al. ESC Guidelines for the management of acute myocardial infarction in patients presenting with ST-segment elevation. Eur. Heart J. 2012, 33, 2569-2619. [CrossRef] 
27. A A Fox, K.; Fitzgerald, G.; Puymirat, E.; Huang, W.; Carruthers, K.F.; Simon, T.; Coste, P.; Monsegu, J.; Steg, P.G.; Danchin, N.; et al. Should patients with acute coronary disease be stratified for management according to their risk? Derivation, external validation and outcomes using the updated GRACE risk score. BMJ Open 2014, 4, e004425. [CrossRef]

28. Cedó, L.; Fernández-Castillejo, S.; Rubió, L.; Metso, J.; Santos, D.; Muñoz-Aguayo, D.; Rivas-Urbina, A.; Tondo, M.; Méndez-Lara, K.A.; Farràs, M.; et al. Phenol-Enriched Virgin Olive Oil Promotes Macrophage-Specific Reverse Cholesterol Transport In Vivo. Biomedicines 2020, 8, 266. [CrossRef] [PubMed]

29. Soria-Florido, M.T.; Schroder, H.; Grau, M.; Fitó, M.; Lassale, C. High density lipoprotein functionality and cardiovascular events and mortality: A systematic review and meta-analysis. Atherosclerosis 2020, 302, 36-42. [CrossRef] [PubMed]

30. Lee-Rueckert, M.; Escola-Gil, J.C.; Kovanen, P.T. HDL functionality in reverse cholesterol transport-Challenges in translating data emerging from mouse models to human disease. Biochim. Biophys. Acta (BBA)-Mol. Cell Biol. Lipids 2016, 1861, 566-583. [CrossRef] [PubMed]

31. Li, X.S.; Wang, Z.; Cajka, T.; Buffa, J.A.; Nemet, I.; Hurd, A.G.; Gu, X.; Skye, S.M.; Roberts, A.B.; Wu, Y.; et al. Untargeted metabolomics identifies trimethyllysine, a TMAO-producing nutrient precursor, as a predictor of incident cardiovascular disease risk. JCI Insight 2018, 3, 3. [CrossRef]

32. Mueller, D.; Allenspach, M.; Othman, A.; Saely, C.H.; Muendlein, A.; Vonbank, A.; Drexel, H.; von Eckardstein, A. Plasma levels of trimethylamine-N-oxide are confounded by impaired kidney function and poor metabolic control. Atherosclerosis 2015, 243, 638-644. [CrossRef] [PubMed]

33. Schiattarella, G.; Sannino, A.; Toscano, E.; Giugliano, G.; Gargiulo, G.; Franzone, A.; Trimarco, B.; Esposito, G.; Perrino, C. Gut microbe-generated metabolite trimethylamine-N-oxide as cardiovascular risk biomarker: A systematic review and dose-response meta-analysis. Eur. Heart J. 2017, 38, 2948-2956. [CrossRef]

34. Yao, M.-E.; Liao, P.-D.; Zhao, X.-J.; Wang, L. Trimethylamine-N-oxide has prognostic value in coronary heart disease: A meta-analysis and dose-response analysis. BMC Cardiovasc. Disord. 2020, 20, 7. [CrossRef] [PubMed]

35. Ibanez, B.; James, S.; Agewall, S.; Antunes, M.J.; Bucciarelli-Ducci, C.; Bueno, H.; Caforio, A.L.P.; Crea, F.; Goudevenos, J.A.; Halvorsen, S.; et al. 2017 ESC guidelines for the management of acute myocardial infarction in patients presenting with STsegment elevation: The task force for the management of acute myocardial infarction in patients presenting with ST-segment elevation of the European Society of Cardiology (ESC). Eur. Heart J. 2018, 39, 119-177. [CrossRef] [PubMed] 